\title{
ESTUDO DO ESTADO NUTRICIONAL DE CRIANÇAS NA IDADE ESCOLAR NA CIDADE DE FRANCA-SP: UMA INTRODUÇÃO AO PROBLEMA
}

\author{
A STUDY OF THE NUTRITIONAL STATE OF SCHOOL CHILDREN IN THE TOWN OF FRANCA, SP: \\ AN INTRODUCTION TO THE PROBLEM
ESTÚDIO DEL ESTADO NUTRICIONAL DE LOS ESCOLARES DE LA CIUDAD DE FRANCA, SP: UMA INTRODUCCIÓN AL PROBLEMA

\section{Maria Aparecida T. Cano ${ }^{1}$, Cláudia Haddad Caleiro Pereira ${ }^{2}$, Carolina Cristina Coimbra Silva ${ }^{3}$, Juliana Neves Pimenta ${ }^{4}$, Paula Silva Maranha ${ }^{5}$}

RESUMO: Dentre os problemas nutricionais da infância, a obesidade é a que mais chama a atenção na área da saúde. Objetivo: avaliar o perfil nutricional de crianças entre 7 e 8 anos de idade em escolas públicas e privadas da cidade de Franca-SP. Metodologia: dividimos as escolas segundo a clientela que as freqüentava em carentes, mistas e particulares. Nossa amostra foi de 171 crianças que foram entrevistadas, pesadas e medidas. Resultado: 44 alunos $(25,6 \%)$ apresentaram um tipo de risco nutricional, sendo $29(16,9 \%)$ com risco para obesidade, e destes, $8(27,5 \%)$ freqüentavam escola carente ou mista. Conclusão: tanto crianças com risco para obesidade, quanto aquelas consideradas "normais" para o peso não têm uma alimentação saudável e vivem uma vida sedentária. As cantinas das escolas não são supervisionadas. Nesta primeira aproximação do tema, verificamos que Franca, nós já temos escolares com risco para obesidade.

PALAVRAS CHAVES: Saúde Escolar; Obesidade; Avaliação Nutricional.

SUMMARY: Among nutritional problems during infancy, obesity is relevant concerning to health state. Objective: evaluate the nutritional profile of children who were 6 and 7 years old in public and private schools in Franca, São Paulo. Methodology: We separated schools according to the group of students that were attending them, in caring, mixed and private schools. Our sample were composed by 171 children that were interviewed, weighed and had their height measured. Results: 44 students $(25,6 \%)$ presented at least one nutritional risk, and $29(16,9 \%)$ with obesity risk, and $8(27,5 \%)$ of these attended caring or mixed school. Conclusion: children with obesity risk and those considered "normal" for weigh do not have a healthy nourishment and live a sedentary life. School canteens are not well supervised. In this first approximation of this theme we verified that in Franca we can detect children with risk for obesity.

KEYWORDS: School Health; Obesity; Nutritional Assessment.
RESUMEN: Dentro de los problemas nutricionales de la infancia, la obesidad es lo que más llama la atención en el area de la salud. Sentimos necesidad de conocer la realidad del estado nutricional de los escolares de la ciudad de Franca, SP. Objetivo: evaluar el perfil nutricional de niños entre 7 y 8 años de edad en escuelas públicas y privadas de la ciudad. Metodología: dividimos las escuelas de acuerdo con la clientela que las frecuentaba en carentes, mixtas y particulares. Nuestra muestra fue de 171 niños que fueron entrevistados, pesados y medidos. Resultado: 44 alumnos $(25,6 \%)$ presentaron un tipo de riesgo nutricional, de los cuales 29 (16,9\%) presentaron riesgo de obesidad, $y$ de estos, $8 \quad(27,5 \%)$ frecuentaban una escuela carente o mixta. Conclusión: tanto los niños con riesgo de obesidad, como aquellos considerados "normales" con respecto a su peso no tienen una alimentación saludable y viven una vida sedentária. Las cantinas de las escuelas no son supervisadas. En esta primera perspectiva del tema, verificamos que en Franca ya hay escolares con riesgo de obesidad.

PALABRAS CLAVES: Salud Escolar; Obesidad; Evaluación Nutricional.

1 Pesquisador da FUNADESP. Orientador do Curso de Pós Graduação de Promoção da Saúde-Universidade de Franca - UNIFRAN. R. Pau Brasil 824 bairro J. Recreio. Ribeirão Preto - SP CEP 14040-220. e-mail: decano@netsite.com.br

2 Professora do Curso de Graduação em Nutrição e Mestranda no Curso de Pós-Graduação de Promoção da Saúde da Universidade de Franca. Rua Dos Pracinhas no 1871 bairro- Residencial Paraíso. Franca-SP CEP - 14403160. e-mail: caleiropereira@uol.com.br

3 Aluna do Curso de Graduação em Nutrição da Universidade de Franca. Rua .Avenida Vigário Paixão n 57 apto. 402 bairro - Centro. Sacramento MG CEP - 38190 000. e-mail: carinacs@bol.com.br

4 Aluna do Curso de Graduação em Nutrição da Universidade de Franca. R. Voluntário José Rufino $n^{\circ} 1269$ bairro - centro. Franca-SP CEP - 14400.580 e- mail: juliana.np@uol.com.br

5 Aluna do Curso de Graduação em Nutrição da Universidade de Franca. R. José Chediack $n^{\circ} 265$ bairro - Vila Monteiro. Franca- SP CEP 14401.141. e-mail: paulamaranha@hotmail.com.br 


\section{INTRODUÇÃO}

Nos últimos anos tem sido muito discutida a questão da obesidade, em todo o Brasil e em diferentes classes sociais. Muitas pesquisas sobre o tema vêm alertando a sociedade para este tipo de problema. (SANTOS, 1997; MONTEIRO et al. 1995; PIZZATTO, 1992).

Ao mesmo tempo, no Brasil ainda se discute o problema da fome que atinge milhões de brasileiros todos os anos, entre homens, mulheres e crianças, e é considerado um grave problema de Saúde Pública.

Assim como a desnutrição, a obesidade especialmente na infância pode trazer sérios danos à saúde global. Segundo MONTEIRO et al. (1995), o Brasil vive um momento de transição nutricional, ou seja, estão ocorrendo mudanças nos padrões alimentares dos indivíduos em conseqüência de modificações em sua dieta decorrente de mudanças sócias, econômicas e influencia da mídia.

Concordamos com PIZZATTO (1992), que a principio o estudo da obesidade pode parecer irrelevante em um país em crise financeira e pessoal, no entanto a obesidade representa um fenômeno de incidência cada vez mais elevada tanto entre as classes desprivilegiadas economicamente, como nas mais abastadas, não apenas no Brasil, mas em todos os países.

A obesidade hoje supera os índices de desnutrição. No Brasil o número de obesos é calculado em 17 milhões de pessoas, ou 9,6 \% da população (PIZZATTO, 1992).

A obesidade na infância pode trazer sérias conseqüências para a saúde como a hipertensão, o colesterol alto, problemas cardíacos, morte prematura, entre outros.

Por outro lado, como citam WHALEY \& WONG (1989), com a evolução da obesidade na criança, na maioria das vezes pode ocorrer sua marginalização pela falta de aceitação das outras crianças consideradas "normais" com relação ao peso. Esta situação leva a criança obesa a ter uma baixa alto estima, como conseqüência, ela se isola socialmente, brinca menos, se sente envergonhada e come em excesso para aliviar o sofrimento e com isso fecha-se o ciclo vicioso.

Mediante a alimentação adequada em quantidade e qualidade, o organismo adquire a energia e os nutrientes necessários para o bom desempenho de suas funções e para a manutenção de um bom estado de saúde. É de se ressaltar o conhecimento dos prejuízos decorrentes quer do consumo alimentar insuficiente (deficiências nutricionais), quer do consumo alimentar excessivo (obesidade) (MONTEIRO et al. 1995).

Diante do exposto, sentimos necessidade de uma primeira abordagem, nos aproximarmos da realidade do estado nutricional das crianças em idade escolar em Franca-SP e concordamos com BARBIERI et al. (1988), que a avaliação do estado nutricional da população infantil é importante aos serviços de saúde pública e aos pesquisadores e que a aferição do peso e da altura permitem a comparação de cada criança com o padrão de crescimento ideal para que se verifique a existência de possíveis deficiências.

\section{OBJETIVO}

Avaliar o perfil nutricional de crianças na faixa etária de 7 a 8 anos de idade de escolas públicas e privadas da cidade de Franca-SP.

\section{METODOLOGIA}

Esta pesquisa é de natureza descritivoexploratória e foi desenvolvida com uma amostra de 171 crianças de 7 a 8 anos de idade, matriculadas em duas escolas públicas e uma privada da cidade de Franca-SP. O projeto de pesquisa foi aprovado pelo Comitê de Ética em Pesquisa da Universidade de Franca-UNIFRAN.

Para a determinação das escolas que participariam da pesquisa utilizamos como referencial a classificação de CANO \& SILVA (1994) que agrupa as escolas em carentes, mistas e particulares de acordo com a clientela que as freqüenta. As escolas carentes recebem crianças provindas de famílias de baixa renda, moradoras em sua maioria em bairros periféricos pobres da cidade. Os pais têm baixa escolaridade e estão empregados em atividades braçais, no comércio, construções civis, na lavoura. As escolas mistas apresentam uma clientela heterogênea com relação ao nível sócio econômica das famílias, geralmente estão localizadas no Centro da cidade e são escolas grandes. As escolas particulares agrupadas nesta classificação são aquelas em que a mensalidade é maior ou igual a 1 salário mínimo, portanto, recebem alunos de melhor nível sócio econômico e cultural.

Entramos em contato com a Delegacia de Ensino de Franca, apresentamos o projeto de pesquisa e seus objetivos e solicitamos permissão para desenvolvê-lo nas escolas. Baseado na classificação de CANO \& SILVA (1994) solicitamos que fosse indicada uma escola de cada grupo. A partir da indicação, entramos em contato com os diretores, agendamos uma reunião e expusemos o projeto de pesquisa.

Após o acerto, os diretores entraram em contato com os pais solicitando autorização para que as crianças pudessem participar. Foi elaborado junto com os diretores um cronograma de datas e horários oportunos para a coleta de dados, dentro do período de aulas. Em cada escola, todas as crianças de primeira série fizeram parte da amostra.

A coleta de dados ocorreu no período de maio a setembro de 2004.

Elaboramos um questionário com questões que levantaram informações sobre o consumo alimentar 
das crianças, especificamente com relação ao lanche, uso da cantina ou merenda escolar e ingestão de refrigerantes e doces; questões relativas a atividades físicas, de esporte e lazer. Neste questionário foram anotadas as avaliações antropométricas de peso e altura para a idade e Índice de Massa Corpórea -IMC.

A coleta de dados desenvolveu-se em três etapas:

Primeira: nesta etapa foi realizada a avaliação antropométrica utilizando-se uma balança (Welmy, ano de fabricação 2004, modelo 110 , número de série 69420, verificação inicial 0059.049-5, peso máximo $150 \mathrm{Kg}$ ). As crianças foram orientadas a tirar o tênis e o moletom para não interferir nos dados. O peso e altura foram anotados no gráfico de crescimento e desenvolvimento, utilizados pela Secretaria Estadual de Saúde de São Paulo, baseado nas curvas de crescimento Classe IV de Santo André (São Paulo, SES, S.d.). Utilizou-se também a Tabela E1 Percentis de IMC $\left(\mathrm{Kg} / \mathrm{m}^{2}\right)$ para avaliação de Estado Nutricional de crianças e adolescentes, sendo considerado:

$\leq \mathrm{P} 5$ baixo peso

P5 - P15 risco para baixo peso

P15 - P85 eutrofia

P85 - P95 sobrepeso

$\geq 95$ obesidade (anexo 3 ).

Durante esta etapa também foram realizadas as entrevistas individuais, para que não houvesse indução de respostas dos colegas.
Segunda: De acordo com os resultados obtidos, foi realizada uma segunda pesagem dos alunos que na primeira saíram do perfil eutrófico e para que houvesse confirmação do dado. Esta etapa ocorreu entre 10 e 15 dias após a primeira pesagem.

Terceira: Foram comparados os resultados da primeira e segunda pesagem e os IMC e os alunos que se apresentaram na faixa de risco tanto para obesidade, como para a desnutrição, foram indicados para os diretores das escolas que agendaram reuniões com os pais para as orientações nutricionais. Cabe salientar que tanto a segunda, como a terceira etapa foi realizada levando-se em considerações os aspectos éticos de proteção especialmente as crianças com risco de obesidade.

\section{RESULTADOS E DISCUSSÃO}

Dos 171 alunos que participaram da pesquisa, 44 ou $25,6 \%$ apresentaram um tipo de risco nutricional, sendo que $15(8,7 \%)$ alunos apresentaram risco para desnutrição e destes apenas um (1) $(6,6 \%)$ pertence à escola particular, os demais estão matriculados em escola mista ou carente.

Com relação à obesidade, 29 alunos ou seja $16,9 \%$ da amostra estão nesta faixa de risco e destes $8(27,5 \%)$ pertencem às escolas mista e carente, conforme mostra o gráfico 1 a seguir:

\section{Gráfico 1}

Distribuição dos alunos por grupo de risco de acordo com o tipo de escola

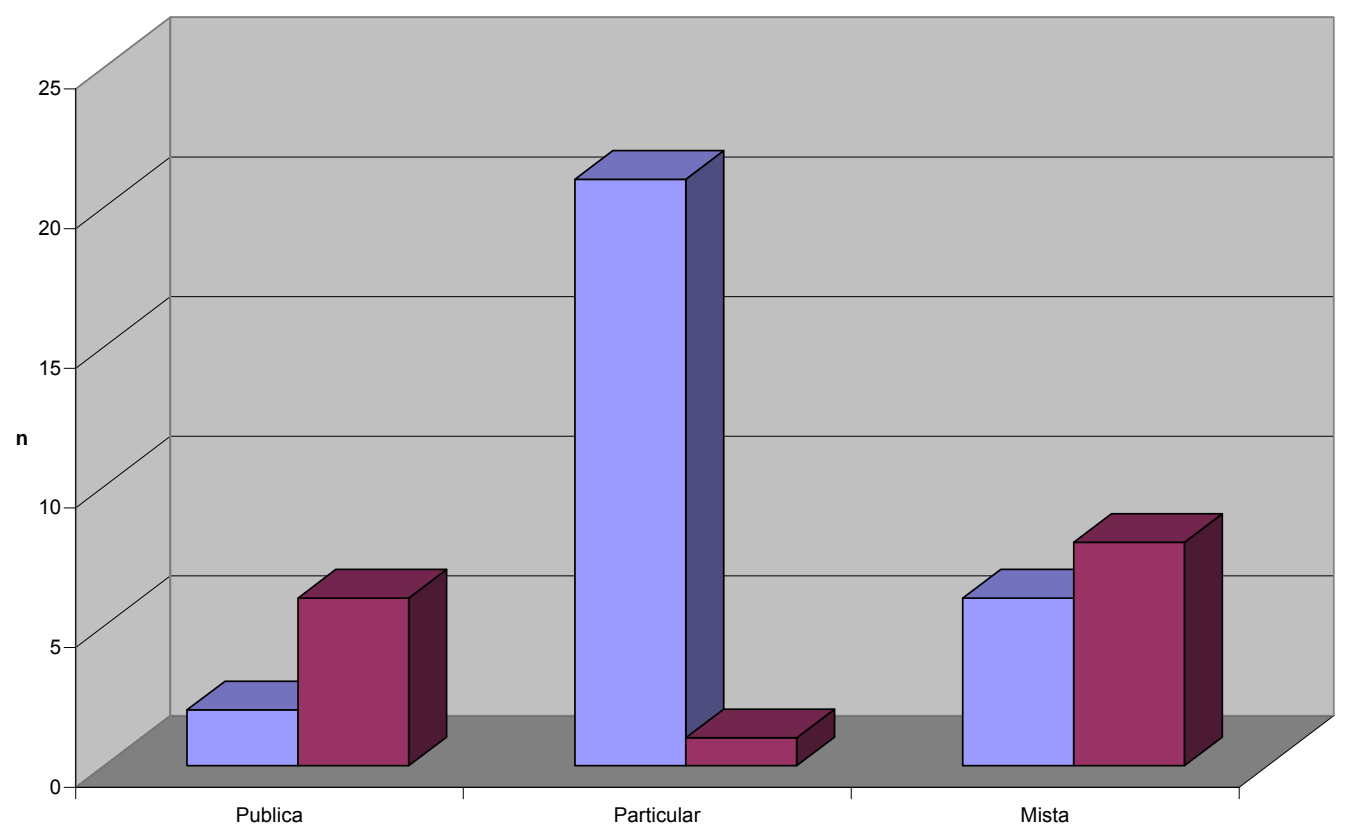


Estudo do estado nutricional de crianças na idade escolar na cidade de franca-sp: uma introdução ao problema. Revista Eletrônica de Enfermagem, v. 07, n. 02, p. 179 - 184, 2005. Disponível em http://www.fen.ufg.br

Os dados da nossa pesquisa apresentam uma tendência que é mundial de um número cada vez maior de pessoas obesas ou com risco para obesidade. A obesidade hoje supera os índices de desnutrição (PIZZATTO, 1992).

Segundo WHALEY \& WONG (1989), em alguns casos foi demonstrado que a hereditariedade é um fator que contribui para o desenvolvimento da obesidade, mas, na maioria dos casos, os padrões alimentares têm uma base cultural e social.

Com relação aos hábitos alimentares, observouse que estes se mostraram inadequados em todas as escolas pesquisadas, havendo um baixo consumo diário de alimentos básicos e importantes para a formação das crianças, como legumes, frutas, cereais e um alto consumo de alimentos hipercalóricos e inadequados à essa faixa etária (salgadinhos, refrigerantes, chocolates, bolachas doces, bolo).

Sabe-se que hábitos alimentares inadequados podem constituir-se em fatores de risco para a presença de doenças crônicas, tanto na vida atual quanto futura (MENDES et al., 2001)

Os hábitos alimentares são precocemente condicionados nas crianças por seus pais e, como atualmente há uma tendência para alimentos mais gordurosos ou calóricos de fast-food, essas crianças podem desenvolver obesidade desde cedo.

Verificamos que as crianças comem ainda muitos alimentos tipos salgadinhos industrializados riquíssimos em gorduras e calorias.

Este estudo nos mostrou o excessivo uso de refrigerantes e sucos industrializados ricos em calorias piorando ainda o problema em questão. Os escolares ingerem atualmente mais do que o dobro da quantidade de refrigerantes do que há 2 décadas (VIUNISK, 2004).

É muito importante que se ensine aos filhos, com idade entre 2 e 12 anos, a importância de se cultivar hábitos alimentares saudáveis. As crianças necessitam de nutrientes importantes para crescer.

Com relação ao horário de lanche na escola, verificamos que todas as crianças utilizam a cantina para a compra de balas, pirulitos, salgados fritos, refrigerantes, entre outros, mesmo que tragam lanche de casa ou comam a merenda escolar.

$\mathrm{Na}$ escola é importante enfatizar a hora do recreio. A função da escola é educar inclusive durante o recreio. As cantinas e merendas devem oferecer opções saudáveis, o que é muito raro.

A escola também tem papel fundamental ao modelar as atitudes e comportamentos das crianças sobre Nutrição. Uma forma de realizar este trabalho é integrar a nutrição à sala de aula, incorporando conceitos de Nutrição às crianças (SCHARTZMAN \& TEIXEIRA, 2001).

Uma das escolas pesquisada,considerada mista tem horta e os legumes plantados são utilizados na merenda escolar. Esta proposta da escola vai ao encontro de um projeto denominado "Escola Saudável" (VIUNISKI, 2004), cujo objetivo é reduzir a obesidade infantil, através supervisão das cantinas, a implantação de hortas e orientação nutricional.

Nos últimos anos, a obesidade aumentou entre crianças e segundo KOCHI (2004) ela é decorrente de fatores genéticos, ambientais e psicossociais. Segundo a autora as crianças de camadas mais pobres da população não têm informação correta sobre os alimentos e as crianças de maiores níveis econômicos sofrem a falta de vínculo mãe-filho decorrente especificamente do trabalho da mãe fora do lar, o que também acarreta erros alimentares.

Observou-se na pesquisa que o lanche preparado em casa pela mãe, ou empregada, é também bastante calórico. Verificou-se que das 44 crianças com risco nutricional, 15 levam bolo recheado, pizza, chocolate ou bolacha com recheio; 9 levam salgadinhos industrializados; 10 levam refrigerantes.

Verificou-se que apenas 6 alunos levam suco natural industrializados ou fruta.

Antigamente os lanches levados de casa eram considerados lanches saudáveis (suco natural, pão com manteiga ou queijo e frutas), mas hoje, com a correria do dia a dia a opção é sempre pelo mais "fácil" ou seja alimentos industrializados.

Outro dado importante citado na pesquisa pelas crianças foi o hábito de se fazer as refeições em casa assistindo a televisão, ou brincando (sempre tem que ter algum brinquedo nas mãos para fazer as refeições) ou jogando vídeo-game, com isso ela não aprende a sentir o sabor e aroma do que está mastigando e muito menos sabendo o que está comendo. É importante que a criança coma vagarosamente, mastigando várias vezes o alimento realizando na boca a primeira digestão. Quando a criança não mastiga adequadamente os alimentos, a quantidade ingerida é maior podendo ocorrer a obesidade.

Com relação à atividade física, encontramos nesta pesquisa, que todos os alunos participam daquela que é obrigatória na escola, sendo excluídos apenas os alunos que apresentam problemas de saúde e são dispensados pelo médico.

Das 44 crianças que apresentaram um tipo de risco nutricional, verificou-se que 16 realizam atividades físicas fora do horário de aula como, natação, futebol, basquete e balet. Encontrou-se, no entanto 28 crianças que consideraram como atividade física, aulas de música, desenho e computação.

A atividade física é fundamental para o desenvolvimento das crianças. Incluir esportes, brincadeiras ao ar livre, passeios e caminhadas no fim de semana. Hoje estas atividades são excluídas em todas as classes sociais em decorrência da violência e riscos de acidentes. Por outro lado é necessário restringir o tempo na frente da TV e de videogame. Temos de pensar que os maus hábitos alimentares associados ao sedentarismo estão levando a uma epidemia de crianças obesas que, se não tratadas, desenvolverão graves problemas físicos e psicológicos. 
Como vimos nesta pesquisa, o índice maior de risco nutricional é a obesidade que aparece em todas as camadas sociais. Segundo CORRÊA (2004), no Brasil, muitas crianças menores de 5 anos ainda apresentam baixo peso e nanismo, mas paralelamente tem surgido um novo problema alimentar no país que é um aumento da obesidade infantil.

Crianças obesas podem fazer parte dos grupos de riscos com maiores probabilidades de virem a sofrer, na idade adulta, de distúrbios tais como a hipertensão, diabetes, doenças respiratórias, etc. (CORRÊA, 2004).

Além disso, a Educação Nutricional é essencial, pois visa a modificação e melhorias dos hábitos alimentares a longo prazo, e torna-se um elemento de conscientização e reformulação das distorções do comportamento alimentar, auxiliando a refletir sobre a saúde e qualidade de vida (MANTOANELLI, et al, 1997).

O Profissional Nutricionista e os profissionais de saúde têm papel relevante na orientação de escolas (merendas e cantinas), junto às famílias na prevenção da obesidade, podendo auxiliar na reversão do quadro acima encontrado.

Para melhores resultados nos tratamentos é importante a cooperação dos pais e educadores que devem estar conscientes de que a obesidade é um risco e que gera problemas na vida adulta.

\section{CONCLUSÃO}

Pelos dados encontrados em nossa pesquisa, embora seja apenas uma primeira abordagem sobre o tema, verificamos que a cidade de Franca-SP, já enfrenta o problema da obesidade infantil. Encontramos que de 171 alunos da rede de ensino, $16,9 \%$ apresentam risco de obesidade em todas as camadas sociais.

As cantinas das escolas, não contam com um sistema de vigilância nutricional, vendendo todo tipo de alimentos industrializados e calóricos, o que aumenta a probabilidade de risco para a obesidade, assim como perpetua os vícios de uma alimentação não saudável mesmo para crianças que não fazem parte do grupo de risco.

Verificou-se que mesmo os lanches trazidos de casa não contemplam uma alimentação saudável.

O sedentarismo das crianças é outro fator que eleva o risco de obesidade.

Os maus hábitos alimentares, o sedentarismo e a ausência de uma vida saudável na infância perpassa todas as camadas sociais e se faz presente também entre as crianças consideradas "normais" para o peso.

Percebe-se que somente a união de pais, professores e profissionais da saúde, em especial o Nutricionista poderão minimizar 0 problema da obesidade.

\section{REFERÊNCIAS BIBLIOGRÁFICAS}

BARBIERI, M.A et al. Crescimento e estado nutricional. In: WOISKI, J.R. Nutrição e dietética em pediatria clínica. Rio de Janeiro, ATHENEU,1988

CANO, M.A.T.; SILVA, G.B da. Detecção de Problemas Visuais e Auditivos de Escolares em Ribeirão Preto: estudo comparativo por nível sócioeconômico . Rev. Latino- Am. Enf., v. 2, n. 1, p. 57-68, 1994.

CORRÊA, N.C. Qual o segmento da população mais afetado? Revista CRN-69. p.9-10. jan 2004.

$\mathrm{KOCHI}, \mathrm{C}$. Escola saudável dá certo. Revista CRN-3. Notícias. Edição n 76. p 32. dez., 2004.

MANTOANELLI, G., COLUCCI, A.C.A; CRUZ, A.T.R., et al. Avaliação de rótulos e embalagens: bebidas lácteas, iogurte,queijo tipo petit suisse na alimentação infantil. In: Simpósio de Iniciação Científica da USP. Ribeirão Preto. 1997.

MENDES, F.S.V.; PRIORE, S.E.; RIBEIRO, S.M.R. et al. Avaliação do estilo de vida e condições nutricionais de adolescentes atendidos em um programa específico. Rev. Nutrição em Pauta, n.2, p.20-24, 2001.

MONTEIRO, C.A. et al. Da desnutrição para a obesidade: a transição nutricional no Brasil. In: MONTEIRO,C.A.(org) Velhos e novos males da saúde no Brasil. São Paulo, Hucitic,1995. p.248-54.

PIZZATTO, V.T. Obesidade infantil. São Paulo, Sarvier,1992. Notícias. Edição n76. Dez 2004.

SANTOS, G.V.B. Desnutrição e obesidade em préescolares da rede Municipal de Ensino de Ribeirão Preto, 1997.151p. Dissertação (Mestrado). Escola de Enfermagem de Ribeirão Preto- Universidade de São Paulo.

SÃO PAULO. Secretaria de Estado da Saúde. Treinamento de multiplicadores nas ações básicas de saúde da criança.São Paulo-SP, s/d. 44p.

SCHARTZMAN, F.; TEIXEIRA, A.C..Educação nutricional prevendo a obesidade. Revista Nutrição em Pauta. n.60, p.19-20, Set 2001.

VIUNISK, N. Projeto Escola Saudável. Associação Brasileira para o Estudo da Obesidade e da Síndrome Metabólica. 2004 [online] Disponível em www.abeso.org.br. [Acesso em 15 mai. de 2004].

WHALEY, L.F; WONG, D.L. Enfermagem Pediátrica. 2ed. Rio de Janeiro, Guanabara Koogan, 1989. 
Estudo do estado nutricional de crianças na idade escolar na cidade de franca-sp: uma introdução ao problema. Revista Eletrônica de Enfermagem, v. 07, n. 02, p. 179 - 184 2005. Disponível em http://www.fen.ufg.br

ARTIGO ORIGINAL 